\title{
Stress as a Workload in Intensive Care Units
}

\author{
E. Zacharova (Eva Zacharova) $)^{1}$, K. Moltasova (Katerina Moltasova)², \\ M. Kyasova (Miroslava Kyasova)²
}

${ }^{1}$ St. Elizabeth University of Health and Social Work, Bratislava, SK.

${ }^{2}$ Department of Nursing and Midwifery, Faculty of Medicine, Masaryk

University, CZ.

\section{E-mail address:}

eva.zacharova@seznam.cz

\section{Reprint address:}

\section{Eva Zacharova}

St. Elizabeth University of Health and Social Work

Bratislava

Slovakia

Source: Clinical Social Work and Health Intervention

Volume: 12

Issue: 3

Pages: 55 - 58

Cited references: 7

\section{Reviewers:}

Jirina Kafkova

MSF, Freetown, SL

Zofia Szarota

Pedagogical University of Cracow, PL

\section{Keywords:}

Stress. Stressors. Intensive Care Workplace. Physical Activity. Mental Stress.

\section{Publisher:}

International Society of Applied Preventive Medicine i-gap

CSWHI 2021; 12(3): 55 - 58; DOI: 10.22359/cswhi_12_3_10 (C) Clinical Social Work and Health Intervention

\section{Abstract:}

Stress is one of the significant workloads in intensive care units. This survey examines the relationship between the level of stress experienced, gender, age, length of practice and the degree of the workplace. It maps the factors that lead to the emergence of stress in intensive care units and examines its impact on work performance. Results from 121 respondents working in intensive care units show that the level of stress correlates with age and is typical for certain stages of life. Work stress is mostly created by load, where the physical and mental component is applied. Total physical activity corresponded to moderate load and the most important physical factors represented increased demands on the musculoskeletal system and disturbance of sleep rhythm. The level of mental stress was also in the moderate values. The most important psychological stressors were identified as a high degree of responsibility and increased demands on cognitive functions. 


\section{Introduction}

Long-term stress is one of the main causes of many chronic diseases. Stress does not cause a specific disease but increases the risk that an individual will develop a disease for which he has a predisposition. It acts on the body either $d i$ rectly or indirectly. Directly in this sense means that it affects the immune, endocrine or nervous system. Indirectly, on the contrary, means that it affects an individual's behavior, which can then lead to damaging to their health, such as an unhealthy lifestyle (smoking, overeating, etc.). Stress has also been proved to affect mental health. In particular, chronic stress can also cause serious mental health problems, such as depression, post-traumatic stress disorder, anxiety states, or burnout syndrome. ${ }^{1}$ Some degree of stress in the work place is a positive incentive for many individuals to perform better but excessive load and chronic stress has a negative effect. Work in healthcare is generally associated with a higher level of stress, which is associated with: high demands on performance; responsibility; ability to make quick decisions; an assumption for high levels of personal commitment. Factors that influence resistance to load include, in particular: biological factors such as health; age; gender; genetic predisposition to perceive level of stress experienced. Individuality is underlined by confidence in one's own strength and the possibility of influencing events in one's surroundings. A very significant source of work stress is interpersonal relationships in the workplace. Positive relationships evoke a sense of support and belonging, while bad relationships are considered one of the stressors. Both of these opposites are then reflected in how the individual responds to other stressors. ${ }^{2}$

\section{Goal}

The aim of the work was to determine the level of stress experienced by respondents in intensive care units, to map the most important physical and mental stressors and to determine their impact on work performance.

\section{Methodology}

To obtain the data, a custom-designed and non-standardized questionnaire containing 22 items was chosen. The respondents were general nurses, general nurses with specialized qualifi- cations and paramedics working in intensive care units of various levels. The degree of stress and perception of physical and mental stressors were assessed using the Likert scale 1 - 5 (1 - no stress, 5 - overload stress). The numerical data was analyzed in IBM SPSS 25 Statistics software. The following statistical methods were used in the analysis: proportions (frequencies) of variables; contingency tables; arithmetic averages; standard deviations of variance (ANOVA); chi-square test; correlation coefficient. The significance level for verifying the hypotheses was set at $a=0.05$.

\section{Results}

The survey was conducted in the period from November 2019 to the end of January 2020 in 2 university hospitals and 1 city hospital. A total of 150 questionnaires were distributed, which were evenly distributed according to the levels of intensive care units (the highest, III. degree of the ICU). The results were processed from 121 respondents, of which 18 respondents $(15 \%)$ were men and $103(85 \%)$ were women. In terms of the position held, 47 (39\%) respondents worked as a general nurse, $60(50 \%)$ as a nurse with specialized qualifications and $14(11 \%)$ as a paramedic.

The first hypothesis tested whether the reported level of stress depends on gender, age, length of work experience of the respondent and the type of intensive care unit. The average stress rate, regardless of any other characteristics, was 3.066 in our sample, which corresponds to the moderate stress rate. Women suffer from higher stress on average (average 3.278) than men (average 3.029), but this difference is not statistically significant (T-test $\mathrm{p}=0.1379)$. There is no linear relationship between age and stress. The lowest levels of stress were reported by respondents aged 40-49 years (average 2.806); the highest by groups between $30-39$ years (average 3.250 ) and 50-59 years (average 3.250). With the exception of the group with the shortest length of work experience (0-5 years), which reported an average stress rate of 3.061; the right-hand rule applies, that the reported stress rate gradually decreases with the increased length of work experience. Respondents with 26 to 30 years of work experience reported an average stress rate of 2.875. This dependence is not statistically significant (ANOVA $\mathrm{p}=0.1669$ ). 
For all types of intensive care units, the average reported stress level was around level 3 which means a medium level of stress. The highest level of stress was reported by respondents in the ICU I workplace (average 3.231); the lowest level of stress was reported by respondents in the ICU II workplace (average 3,120 ) but the differences were not statistically significant (ANOVA $\mathrm{p}=0,8430)$.

The second hypothesis tested the relationship between the level of stress in the workplace and physical stressors. A Summation Index was created, which showed how many stress factors the respondent stated. Higher numbers meant that the respondent was stressed by several factors at the same time. Furthermore, the correlation coefficient between the number of stress factors and the degree of physical activity was calculated. Pearson's coefficient $r=0,2448$ indicated a slight but pre-existing dependence. As the number of stress factors increased, so did the level of reported physical activity. A similar approach was chosen to determine the relationship between information on perceived stress levels and reported levels of physical activity. Pearson's correlation coefficient $r=0.1756$ indicated a weak but existing dependence. Since both correlations were statistically significant, there is a statistically significant relationship between the level of stress in the workplace and physical stressors.

The third hypothesis tested the relationship between the level of stress experienced by respondents in the workplace and psychological stressors. A Summation Index was also created, which showed how many psychological stressors the respondent stated. Furthermore, the correlation coefficient between the number of psychological stress factors and the degree of psychological stress was calculated. Pearson's correlation coefficient $r=0.2644$ indicated a slight but pre-existing dependence. As the number of stress factors increased, so did the level of reported psychological stress. A similar approach was chosen to determine the relationship between information on perceived stress levels and reported levels of mental strain. Pearson's correlation coefficient $r=0.5719$ indicated a strong dependence. Since both correlations were statistically significant, there is a statistically significant relationship between stress levels and psychological stressors.

\section{Discussion}

$50(41 \%)$ respondents in our sample stated excessive stress; 46 (38\%) respondents stated marginal stress levels; $8(7 \%)$ reported moderate stress levels; 12 (10\%) feel mild stress; 5 (4\%) stated that they do not feel any stress. Svobodová ${ }^{3}$ also states in her bachelor's thesis that work in intensive care units is stressful, where $93.9 \%$ of the 83 nurses interviewed who work in intensive care units are under the influence of stress. Clearly most respondents, 82 (68\%) of our sample, stated that the share of both stressors (physical and mental) is in balance; $30(25 \%)$ stated that mental stress is predominant; $9(7 \%)$ stated the physical stress as being dominant.

Statistical testing confirmed only the relationship between age and stress levels. The relationship between age and stress was also addressed in a foreign study from 2016, which included a total of 82 employees (nurses and doctors) working in intensive care units. It has been shown that ICU staff under the age of 35 have a high prevalence of stress compared to staff over the age of 35.4 These results could to a large extent correlate with the experience gained by nurses during their professional lives. The relationship between gender, type of intensive care unit and stress level could not be confirmed. The greatest physical stress for respondents is a disruption of sleep rhythm - $80(23 \%)$. The second most common response was increased demands on the musculoskeletal system - 78 (22\%), especially joints, muscles and spine. And the third most frequently reported response was that they do not have enough time to eat regularly - 68 (19\%). Vévoda et al. state that completing night shifts demonstrably disrupts physical and mental processes in the body.

Sleep deprivation, as a physical stressor, is also one of the main causes of mental health problems, especially the emergence of anxiety. ${ }^{5}$ There were $66(17 \%)$ respondents, who reported that the greatest mental stress in intensive care units was due to a a high degree of responsibility. $48(13 \%)$ respondents reported that the second largest mental stress is an increase in the requirements for cognitive functions, especially for memory and concentration.

The mental stress of working under time pressure was reported as being the same - $48(13 \%)$ respondents. The relationship between psycho- 
logical stressors and the increasing level of perceived stress was confirmed. In the research part of her work, Svobodová states that nurses in acute medical units perceive the greatest cause of work stress to be in workplace conflicts and in interpersonal relationships. Especially in bad relationships at the level of nurse - doctor and nurse - nurse. ${ }^{3}$ In our sample, $58 \%$ of respondents stated that they had already experienced signs of work overload; $35 \%$ stated that signs of work overload occurred only occasionally; $7 \%$ indicated that they have not yet shown any signs of work overload.

The most common manifestations of work overload include exhaustion - 83 (17\%). The second most common manifestation is lack of concentration - $59(12 \%)$ and forgetfulness - 59 (12\%). Most respondents - 75 (62\%) stated that they sometimes feel that they are more demanding than they are able to handle, 39 (32\%) of respondents do not feel that they are more demanding than they would be able to they were able to handle. Most respondents - 75 (62\%), stated they ocassionally feel that greater demands are placed on them than they are able to handle; 39 (32\%) respondents do not feel that greater demands are placed on them than they would be able to handle. Only 7 (6\%) respondents feel that greater demands are placed on them than they are able to handle. When there is a disproportion between the demands placed and the ability to manage them, it leads to internal tension in the individual and a critical disruption of homeostasis..$^{5}$ Our survey revealed that the vast majority encounter this feeling only occasionally. This fact could also be due to the sudden nature of the work which is typical for intensive care units.

\section{Recommendations for practice}

Prevention of stress factors in the work environment can be divided into 3 basic levels: the first level is leadership (employee management) for leadership positions; the second level takes place at the level of the organization; the third level represents the level of the individual. The art of a team manager is to create an environment in which employees are able to perform at their best, are not under pressure and see meaning in their work. Modern research shows that in such a work environment, people are satisfied..$^{6,7}$

\section{Conclusion}

It is evident that stress cannot be completely avoided in everyday life and working life, and it is also understandable that there are professions in which the level of stress is higher than in others. Studies clearly show that the higher an individual's responsibility, the higher the level of stress he or she feels. A healthy work environment with an acceptable level of stress, with care for mental and physical health, should be one of the main priorities of all organizations.

\section{References}

1. KRIVOHLAVY J (2009) Psychology of Health 3rd ed., Prague, Czech Republic: Portal. P.184.

2. KOCIANOVA R (2010) Personnel Activities and Methods of Personnel Work. Prague, Czech Republic: Grada Publishing. pp.184185.

3. SVOBODOVA A (2018) The Influence of Stress on Nurse's Work [bachelor's thesis]. Prague, Czech Republic: Charles University, 3rd Faculty of Medicine.

4. KUMAR A, PORE P, GUPTA S, WANI AO (2016) Level of stress and its determinants among Intensive Care Unit staff. Indian $J$ Occup Environ Med. 20 (3): pp. 129-132. doi: 10,4103 / 0019-5278,203137

5. VEVODA J, VEVODOVA S, NAKLADALOVA M (2019) Psychosocial Risk in Health Care. Cas Lek Cesk.157. pp. 411-418.

6. LYUBOMIRSKY S, KING L, DIENER E (2005) The benefits of frequent positive affect: Does happiness lead to success? Psychological Bulletin 131 (6). pp. 803-855. https://doi.org/10.1037/0033-

2909.131.6.803.

7. STAW BM, SUTTON RI, PELLED LH (1994) Employee Positive Emotion and Favorable Outcomes at the Workplace. Organ Sci. 5(1): pp. 51-71. 